\section{Pruning the dendritic tree}

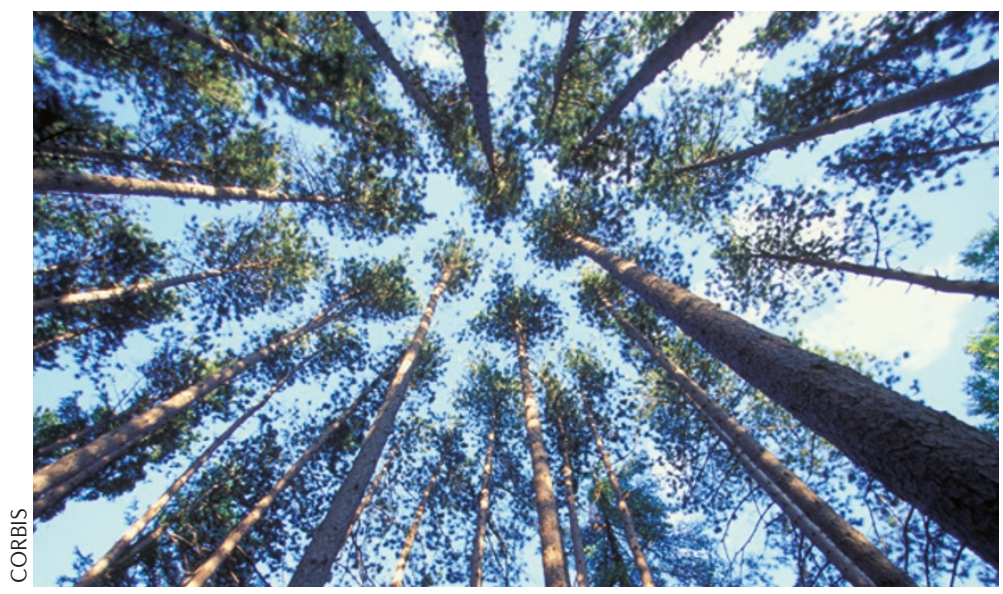

During neural development, the elaboration of dendritic trees is followed by selective pruning - an essential stage in the formation of neural circuits. The mechanisms underlying the switch from dendritic outgrowth to retraction and pruning are unknown, but Puram et al. now suggest that calcium/calmodulindependent kinase type II subunit $\beta$ $(\mathrm{CaMKII} \beta$ ) plays a key part in this process by regulating dendritic patterning via the centrosome.

To characterize the role of CaMKII $\beta$ during the dendritic elongation and pruning phase of development, the authors used RNA interference (RNAi) to acutely decrease CaMKII $\beta$ expression. Surprisingly, they found that this increased total dendrite length and the degree of arborization and reduced the number of dendrite retraction events in cultured cerebellar granule neurons, organotypic cerebellar slices and in vivo cerebellar cortex. These findings suggest that CaMKII $\beta$ is involved in restricting the length of dendrites during development. A similar effect was obtained by knockdown of CaMKII $\beta$ in hippocampal neurons and cerebrocortical neurons, suggesting that CaMKII $\beta$ could have a similar role in various neuronal cell populations.

The function of the carboxyterminal segment of the variable region of CaMKII $\beta$ (CTRv) is unknown, but the authors found that disrupting this domain resulted in effects on dendritic patterning that were similar to those seen after CTRv with green fluorescent protein (GFP) showed that fluorescent puncta colocalized with pericentrin, a centrosomal protein. Indeed, biochemical fractionation experiments showed that CaMKII $\beta$ cofractionates with several centrosomal proteins. Collectively, these results suggest that the centrosome might be the subcellular site at which CaMKII $\beta$ exerts its influence on dendritic patterning and that the CTRv is a centrosomal targeting sequence (CTS) that localizes CaMKII $\beta$ to this region. Importantly, the CTS is unique to the $\beta$ isoform knocking down CaMKII $\beta$. Labelling of CaMKII. Accordingly, CaMKII $\beta$ but not CaMKIIa was localized at the centrosome and CaMKII $\beta$ operated in a CaMKIIa-independent manner to promote dendritic patterning.

Using protein-protein association techniques, the authors determined that CaMKII $\beta$ forms a complex with the centrosomal trafficking protein pericentriolar material 1 (PCM1) in a CTS-dependent manner. Knockdown of PCM1 reduced centrosomal targeting of CaMKII $\beta$ and produced a substantial increase in dendritic arborization in granule neurons, which suggests that the ability of CaMKII $\beta$ to control dendritic patterning relies on PCM1-dependent localization of CaMKII $\beta$ to the centrosome.

What is the protein substrate for the catalytic activity of CaMKII $\beta$ at the centrosome? A prime candidate is the mitotic E3 ubiquitin ligase CDC20-anaphase-promoting complex/cyclosome (APC/C), as it has a role in dendritic growth and is localized at the centrosome, and CDC20 contains potential CaMKII $\beta$ phosphorylation sites. The authors determined that phosphorylation of CDC20-APC/C at Ser51 by CaMKII $\beta$ is key to the influence of CaMKII $\beta$ on dendritic patterning. Crucially, in granule neurons, phosphorylation of CDC20-APC/C initiated its dispersion from the centrosome, an effect that was lost on deletion of the CTRv targeting sequence. This dispersion appears to be crucial to the dendritic retraction effects of CaMKII $\beta$ because if CDC20-APC/C is forcibly located to the centrosome, the pruning effect is lost. These experiments suggest a novel role for CaMKII $\beta$ in the control of dendritic patterning.

Sian Lewis

ORIGINAL RESEARCH PAPER Puram, S. V. et al. $A C a M K I I \beta$ signaling pathway at the centrosome regulates dendrite patterning in the brain. Nature Neurosci. 14, 973-983 (2011) 